\title{
Intervalence Band Stark Effect of the Special Pair Radical Cation in Bacterial Photosynthetic Reaction Centers
}

\author{
Thomas P. Treynor, Steven S. Andrews, and Steven G. Boxer* \\ Department of Chemistry, Stanford University, Stanford, California 94305-5080
}

Received: April 17, 2003; In Final Form: July 30, 2003

\begin{abstract}
The Stark spectrum of the intervalence band of the special pair radical cation in bacterial photosynthetic reaction centers is presented. This spectrum, centered at $2600 \mathrm{~cm}^{-1}$, is modeled using a general treatment of intervalence band Stark effects based on a two-state vibronic coupling model of mixed-valency. The observed line shape can be predicted using values for the electronic coupling, driving force, and reorganization energy along a single antisymmetric mode as derived from previous measurements. The model can be used to estimate the difference between the electric dipole moments of the two hole-localized electronic states of a mixedvalence complex, $\Delta \mu_{\mathrm{CT}}$, even if the dipole strength of its IVB is unknown or affected by intensity borrowing by other electronic transitions. We determine the value of $\Delta \mu_{\mathrm{CT}}$ to be $33 \pm 5 \mathrm{D} / f$ for the special pair radical cation in wild-type Rhodobacter sphaeroides reaction centers, where $f$ is the scalar approximation to the local field correction tensor. This value of $\Delta \mu_{\mathrm{CT}}$ is less than would be predicted from the separation between the magnesium ions of the special pair, yet large enough to suggest that the observed dipole strength of its intervalence band is strongly affected by intensity borrowing.
\end{abstract}

The initial electron transfer reaction in bacterial photosynthesis follows the electronic excitation of a strongly interacting pair of bacteriochlorophylls known as the special pair, or P, within the reaction center (RC). ${ }^{1}$ The $\mathrm{RC}$ consists of three polypeptides, denoted $\mathrm{L}, \mathrm{M}$, and $\mathrm{H}$, which bind six bacteriochlorins in a roughly $C_{2}$-symmetric arrangement. $\mathrm{P}$ is comprised of two bacteriochlorophylls, denoted $\mathrm{P}_{\mathrm{L}}$ and $\mathrm{P}_{\mathrm{M}}$ because their magnesium ions are ligated by histidine residues on the $\mathrm{L}$ and $\mathrm{M}$ polypeptides, respectively. They interact strongly to yield both a red-shifted absorption and an increased oxidation potential compared to those of monomeric bacteriochlorophyll. Electron transfer from ${ }^{1} \mathrm{P}$ to only one of two possible bacteriopheophytin acceptors forms $\mathrm{P}^{+}$with a time constant of about 3 ps at room temperature. An understanding of the rates of charge separation and recombination which lead to efficient, unidirectional electron transfer in the RC requires an understanding of the electronic structure of both ${ }^{1} \mathrm{P}$ and $\mathrm{P}^{+}$.

$\mathrm{P}^{+}$is a mixed-valence complex (MVC) with a significant electronic interaction between two nearly degenerate, holelocalized electronic states, $\psi_{\mathrm{P}_{\mathrm{L}}{ }^{+} \mathrm{P}_{\mathrm{M}}}$ and $\psi_{\mathrm{P}_{\mathrm{L}} \mathrm{P}_{\mathrm{M}}}{ }^{+}{ }^{2}$ The hole is said to be either localized on one-half of the dimer or delocalized across the whole dimer to an extent that depends on the degree of mixing between these states. ${ }^{3}$ One common measure of this mixing is the percentage localization of the hole on $\mathrm{P}_{\mathrm{L}}, \% L$. Relative $\mathrm{P}_{\mathrm{L}^{-}}$and $\mathrm{P}_{\mathrm{M}^{-}}$associated spin densities estimated from electron paramagnetic resonance spectra suggest that $\% L$ is approximately 70 for wild-type (WT) RCs on the nanosecond time scale. ${ }^{4}$ Bacteriochlorophyll 9-keto stretching frequencies from FT-Raman spectra of WT and mutant RCs suggest a similar value of $\% L$ on the femtosecond time scale. ${ }^{5}$ These measurements have provided a benchmark for further investigation into the interaction between $\psi_{\mathrm{P}_{\mathrm{L}}{ }^{+} \mathrm{P}_{\mathrm{M}}}$ and $\psi_{\mathrm{P}_{\mathrm{L}} \mathrm{P}_{\mathrm{M}}}{ }^{+}$by constraining the values of the electronic coupling $V_{0}$, driving force

* Corresponding author: $\mathrm{Ph}$ (650) 723-4482; Fax (650) 723-4817; e-mail Sboxer@stanford.edu.
$\Delta \bar{v}$, and reorganization energies $\lambda$ which affect the value of $\% L^{2,6}$

A measurement of the difference between the electric dipole moments of these states, ${ }^{7} \Delta \mu_{\mathrm{CT}}$, would provide a complementary benchmark for further investigation into the electronic structure of $\mathrm{P}^{+}$by constraining other factors. ${ }^{8}$ Given the measured value of $\% L$, a value of $\Delta \mu_{\mathrm{CT}}$ for $\mathrm{P}^{+}$can be estimated from a measurement of the dipole strength of its intervalence band (IVB), a unique vibronic transition in some oxidized RCs that is absent in spectra of monomeric bacteriochlorophyll cations. ${ }^{9,10}$ If it is assumed that $\psi_{\mathrm{P}_{\mathrm{L}}}{ }^{+} \mathrm{P}_{\mathrm{M}}$ and $\psi_{\mathrm{P}_{\mathrm{L}} \mathrm{P}_{\mathrm{M}}}{ }^{+}$interact with each other exclusively, then the dipole strength of the IVB, $m^{2}$, is expected to be related to the values of $\% L$ and $\Delta \mu_{\mathrm{CT}}$ according to ${ }^{11}$

$$
m^{2}=\frac{\% L}{100}\left(1-\frac{\% L}{100}\right) \Delta \mu_{\mathrm{CT}}^{2}
$$

Using this equation and the value of $25 \pm 8 \mathrm{D}^{2}$ that Breton and co-workers ${ }^{9}$ have measured for $m^{2}, 70 \%$ localization of the hole suggests a value of $\Delta \mu_{\mathrm{CT}}$ equal to $11 \mathrm{D}$. This value corresponds to a hole transferred over $2.3 \AA$, which is $28 \%$ as large as the $8.0 \AA$ distance between the magnesium ions of $\mathrm{P}_{\mathrm{L}}$ and $\mathrm{P}_{\mathrm{M}} \cdot{ }^{12}$ The difference between these values might be attributed to a number of effects in MVCs which are recognized to perturb the values of $\Delta \mu_{\mathrm{CT}}$ from estimations provided by metal-metal separation distances. ${ }^{13}$ However, it has been suggested that

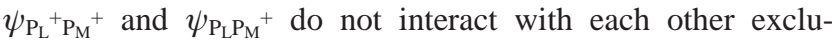
sively, ${ }^{14,15}$ in which case the observed dipole strength might be influenced by intensity borrowing by other electronic transitions in oxidized RCs and thus by factors besides $\% L$ and $\Delta \mu_{\mathrm{CT}}$. In this case the value of $\Delta \mu_{\mathrm{CT}}$ determined using eq 1 is unreliable.

A method of determining the value of $\Delta \mu_{\mathrm{CT}}$ that does not rely on the intensity of the IVB should not only help to quantify the extent of the effects that decrease the effective chargetransfer distance from $8.0 \AA$ but also help to quantify the extent of intensity borrowing. Because electric dipole moments interact 
with electric fields, Stark spectroscopy can be used to estimate the values of $\Delta \mu_{\mathrm{CT}}$ for charge-transfer transitions. ${ }^{13}$ A Stark spectrum, $\Delta A$, is the change in an absorption spectrum under the influence of an applied electric field, $\vec{F}$ :

$$
\Delta A=A(F \neq 0)-A(F=0)
$$

When $F$ perturbs only an absorber's transition dipole moment and peak position, $\Delta A$ for an isotropic sample is predicted to be equal to a sum of the $\bar{v}$-weighted derivatives of $A(F=0){ }^{16}$ This prediction suggests a common mode of analysis of Stark spectra which we call the classical Stark analysis: Stark spectra recorded at two or more values of the angle $\chi$ between the direction of the field and the polarization of the probing light beam are fit to a sum of the $\bar{v}$-weighted derivatives of the absorption spectrum; the coefficients of the second-derivative components are then used to determine $\Delta \mu$, the magnitude of the linear response of the peak position of an individual absorber to the field, and $\xi$, the angle between $\Delta \vec{\mu}$ and $\vec{m}$.

This classical Stark analysis has been used to characterize the IVSEs of a number of MVCs ${ }^{13,17-20}$ since $\mathrm{Oh}$ and Boxer measured the IVSE of the Creutz-Taube ion in 1989. In some cases the fit values of $\Delta \mu$ have been assumed equal to differences between the dipole moments of ground and excited electronic states of the MVCs. This assumption is justified according to a two-state electronic coupling model of mixed valency, in which these ground and excited electronic states have a difference in dipole moment, $\Delta \mu_{12},{ }^{21}$ whose value is constrained by the values of $\Delta \mu_{\mathrm{CT}}$ and $m$ according to ${ }^{22}$

$$
\Delta \mu_{\mathrm{CT}}^{2}=\Delta \mu_{12}^{2}+4 m^{2}
$$

However, this assumption is not justified by a two-state vibronic coupling model of mixed valency, such as that of Piepho, Krausz, and Schatz (PKS). ${ }^{23}$ In the context of a vibronic coupling model, $\Delta \mu_{12}$ is a function of nuclear configuration, $q$, due to the breakdown of the Born-Oppenheimer approximation between the limits of full delocalization ( $\% L$ equal to 50$)$ and full localization ( $\% L$ equal to either 0 or 100). Thus, in the case of a $70 \%$ localized hole, $\Delta \mu$ as determined by a classical Stark analysis of an IVSE cannot be thought equivalent to $\Delta \mu_{12}(q)$ since the latter is not single valued. Born-Oppenheimer violation in some MVCs should also lead to a significant dependence of the IVB line shape upon field strength, violating a principal assumption underlying the classical Stark analysis. ${ }^{24}$ Some MVCs may also experience significant field-modulated population effects, ${ }^{25}$ violating another principal assumption underlying the classical Stark analysis. Nevertheless, even if the fit value of $\Delta \mu$ for the IVSE of $\mathrm{P}^{+}$were a good approximation to its mean value of $\Delta \mu_{12}(q)$, the possibility of significant intensity borrowing in this case invalidates using eq 3 and the observed values of $\Delta \mu$ and $m$ to determine $\Delta \mu_{\mathrm{CT}}$.

A recent vibronic coupling treatment of intervalence band Stark effects (IVSEs) ${ }^{24}$ provides a different way to determine $\Delta \mu_{\mathrm{CT}}$ from Stark spectroscopy. Because the electronic structure of $\mathrm{P}^{+}$has been characterized by many other techniques, the IVSE of $\mathrm{P}^{+}$is a good case study for demonstrating the utility of this new treatment. To familiarize the reader with the relevant concepts from this treatment of IVSEs ${ }^{24}$ and to justify its use in some cases of significant intensity borrowing, we begin with a discussion of the theoretical foundations underlying this new treatment. We then present the IVSE of the special pair radical cation from Rhodobacter sphaeroides RCs, a measurement that has been made possible by the recent development of methods for recording Stark spectra using an FTIR instrument. ${ }^{26}$ We

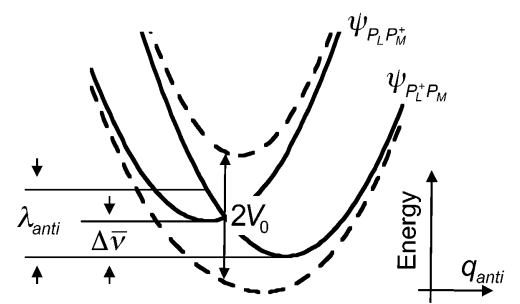

Figure 1. Diabatic surfaces (solid) corresponding to hole-localized

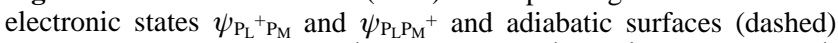
calculated for $V_{0}=1100 \mathrm{~cm}^{-1}, \Delta \bar{v}=600 \mathrm{~cm}^{-1}$, and $\lambda_{\text {anti }}=1100 \mathrm{~cm}^{-1}$.

demonstrate that the observed line shape can be predicted using values consistent with some previously estimated for the electronic coupling, driving force, and reorganization energy along a single antisymmetric mode. We conclude by using the magnitude of the observed IVSE to estimate the value of $\Delta \mu_{\mathrm{CT}}$ for $\mathrm{P}^{+}$

\section{Theoretical Foundations}

When the electronic structure of $\mathrm{P}^{+}$is treated with a vibronic coupling model, for each coupled vibrational mode we must consider its frequency $\bar{v}$ and reorganization energy $\lambda .{ }^{23}$ Although the earliest vibronic coupling models of MVCs considered only antisymmetric modes, with frequencies $\bar{\nu}_{\text {anti }}$ and reorganization energies $\lambda_{\text {anti }}$, it is now agreed that symmetric modes, with frequencies $\bar{\nu}_{\text {sym }}$ and reorganization energies $\lambda_{\text {sym }}$, can become more important than antisymmetric modes as mixing increases. ${ }^{6}$ Using a vibronic coupling model which incorporates only a single antisymmetric mode, Reimers and Hush have shown that $V_{0}=1100 \mathrm{~cm}^{-1}, \Delta \bar{v}=600 \mathrm{~cm}^{-1}$, and $\lambda_{\text {anti }}=1100 \mathrm{~cm}^{-1}$ are values consistent with most measurements of $\mathrm{P}^{+}$in wild-type RCs, including the estimate of $70 \%$ hole localization. ${ }^{6}$ Johnson et al. have recently suggested a different set of values based on an overlapping set of measurements: $V_{0}=1200 \mathrm{~cm}^{-1}, \Delta \bar{v}=$ $900 \mathrm{~cm}^{-1}$, and $\lambda_{\text {anti }}=1800 \mathrm{~cm}^{-1}(\% L \approx 80) .{ }^{11,27}$ Both sets of values make $\mathrm{P}^{+}$a case of intermediate mixing and significant Born-Oppenheimer violation.

Using the former set of values Figure 1 illustrates the diabatic potential energy surfaces corresponding to the hole-localized states $\psi_{\mathrm{P}_{\mathrm{L}}}{ }^{+} \mathrm{P}_{\mathrm{M}}$ and $\psi_{\mathrm{P}_{\mathrm{L}} \mathrm{P}_{\mathrm{M}}}{ }^{+}$as well as the adiabatic potential energy surfaces which result from the $q$-dependent diagonalization of the complete Hamiltonian projected onto this diabatic basis. In this way many of the parameters in this vibronic coupling problem can be easily visualized: $\Delta \bar{v}$ is equal to the energy difference between the minima of the diabatic surfaces, $V_{0}$ is equal to one-half of the splitting of the adiabatic surfaces at the nuclear configuration where the two diabatic surfaces intersect, and $\lambda_{\text {anti }}$ is equal to the difference between the energies of a single diabatic surface evaluated at the nuclear configurations corresponding to the minima of the two diabatic surfaces.

Figure 2 illustrates potential energy surfaces for three different MVCs and their absorption spectra calculated using the PKS model of intervalence band absorption. In each case the values of $V_{0}$ and $\lambda_{\text {anti }}$ have both been set to $1100 \mathrm{~cm}^{-1}$ as in Figure 1 . What distinguishes each pair of surfaces from the other is that the value of $\Delta \bar{v}$ increases from 0 to 600 to $1200 \mathrm{~cm}^{-1}$. To calculate the corresponding spectra, the value of $\bar{\nu}_{\text {anti }}$ has been set to $1200 \mathrm{~cm}^{-1}$, the value employed by Reimers and Hush in their studies. We have performed the calculation at $77 \mathrm{~K}$ and have broadened the stick spectra that result from diagonalizing the complete vibronic Hamiltonian with a Gaussian with full width at half-maximum equal to $700 \mathrm{~cm}^{-1}$. In each case we have used the same value of $\Delta \mu_{\mathrm{CT}}$; however, the line shape of 


$$
\Delta \bar{v}=0 \mathrm{~cm}^{-1}
$$
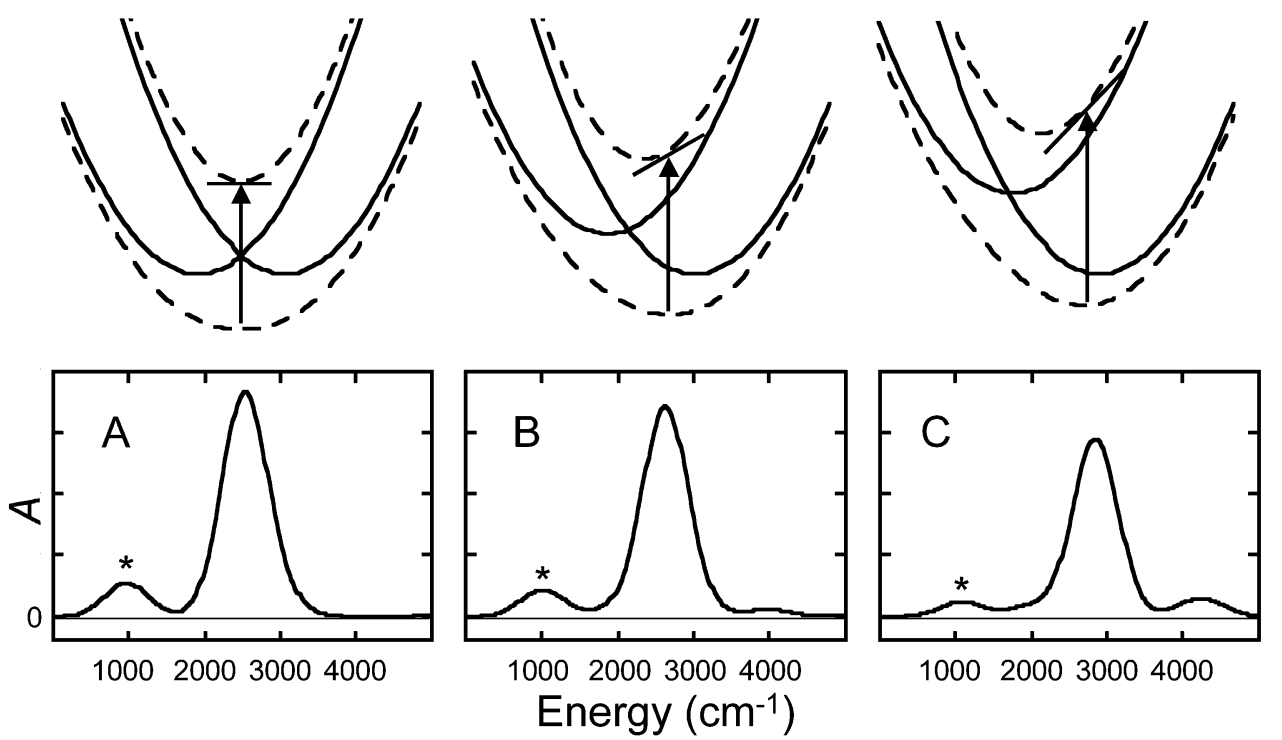

1000200030004000

Figure 2. Simulated absorption spectra and surfaces for mixed-valence complexes with $\Delta \bar{v}$ equal to $0 \mathrm{~cm}^{-1}$ (panel $\mathrm{A}$ ), $600 \mathrm{~cm}-1$ (panel B), and $1200 \mathrm{~cm}^{-1}$ (panel C). In each case the values of $V_{0}$ and $\lambda_{\text {anti }}$ are both $1100 \mathrm{~cm}^{-1}$. Spectra are calculated as described in the text. Vertical arrows superimposed upon the surfaces indicate estimates of the peak absorption frequency using the Franck-Condon principle. According to this principle, the illustrated tangents to the upper adiabatic surfaces have different slopes which correspond to the different line widths of the spectra. In each panel the phase-phonon band is indicated with an asterisk.

the IVB is insensitive to this particular value, so we have removed the units from the vertical axes to emphasize this fact.

In Figure 2 the peak position of the IVB is observed to increase from approximately $2500 \mathrm{~cm}^{-1}$ to nearly $3000 \mathrm{~cm}^{-1}$ as $\Delta \bar{v}$ increases from 0 to $1200 \mathrm{~cm}^{-1}$. A satellite band near $4000 \mathrm{~cm}^{-1}$ is observed to increase in intensity as $\Delta \bar{v}$ increases, effectively broadening the IVB. ${ }^{28}$ (The majority of the width of the main band is due to the $700 \mathrm{~cm}^{-1}$ value of the full width at half-maximum of the Gaussian broadening function.) The separation of this band from the main band remains close to the value of $\bar{\nu}_{\text {anti }}$ if this parameter is varied. Although it is not obvious from the spectra in Figure 2, it is also true that the integrated dipole strength decreases as $\Delta \bar{v}$ is increased; this would become more apparent if $\Delta \bar{v}$ were increased further. Each of these qualitative characteristics is predicted by applying the Franck-Condon principle to the adiabatic surfaces at the nuclear configuration corresponding to the minimum of the ground adiabatic surface. ${ }^{24}$ However, each of the absorption spectra in Figure 2 has an additional absorption band, marked with an asterisk, near the value of $\bar{\nu}_{\text {anti. }}$ This band has been referred to as a phase-phonon band, ${ }^{6}$ and its presence reflects an important failure of the Franck-Condon principle in this case of BornOppenheimer violation.

Using Figures 2 and 3, one can make some qualitative predictions as to the line shape of the IVSE for an isotropic ensemble of $\mathrm{P}^{+}$-containing $\mathrm{RCs}^{24}$ Working in the diabatic representation an applied electric field is considered to interact with $\Delta \vec{\mu}_{\mathrm{CT}}$, the nuclear coordinate-independent difference dipole

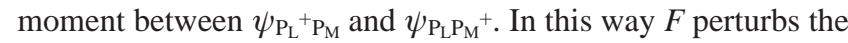
value of $\Delta \bar{v}$ from its value in the absence of the field:

$$
\Delta \bar{\nu}(\vec{F})=\Delta \bar{\nu}-\vec{F} \cdot f \Delta \vec{\mu}_{\mathrm{CT}}
$$

where $f$ is the scalar approximation to the local field correction tensor. This tensor accounts for a possible difference between $\vec{F}$, the externally applied field, and $\vec{F}_{\text {int }}$, the internal field at the position of a chromophore: $\vec{F}_{\text {int }}=f \cdot \vec{F}$. It is generally believed that for most frozen organic or aqueous glasses the value of $f$ should be between 1.0 and $1.3 .^{13,16}$ If the value of $\Delta \mu_{\mathrm{CT}}$ corresponds to the full $8.0 \AA$ between magnesium ions in the special pair and $f$ is presumed equal to 1.3 , an electric field of $0.72 \mathrm{MV} / \mathrm{cm}$ (the largest field obtained in our experiments) will yield a value of $F \cdot f \Delta \mu_{\mathrm{CT}}$ equal to $600 \mathrm{~cm}^{-1}$. Thus, if Figure $2 \mathrm{~B}$ represents the surfaces of $\mathrm{P}^{+}$when $F=0$, then, in the case where $F \cdot f \Delta \mu_{\mathrm{CT}}=600 \mathrm{~cm}^{-1}$, parts A and $\mathrm{C}$ of Figure 2 illustrate the surfaces for the two orientational subpopulations defined by $\Delta \vec{\mu}_{\text {Cт }}$ parallel and antiparallel to $\vec{F}$, respectively.

Figure 3B illustrates the sum of the IVBs of these two orientational subpopulations when $F=0$ (solid line) and their individual IVBs when $F \cdot f \Delta \mu_{\mathrm{CT}}=600 \mathrm{~cm}^{-1}$ (dashed and dotted lines), and we have here treated the transition dipole moment vector as a scalar. A Stark spectrum can then be calculated as the solid line subtracted from the sum of the dashed and dotted lines (eq 2). Its line shape would be very similar to the one illustrated in Figure 3E, which we have instead calculated in a way that is directly comparable with Stark spectra of isotropic samples, using the population-weighted IVBs of 11 orientational subpopulations defined by the angle between $\vec{F}$ and $\Delta \vec{\mu}_{\mathrm{CT}}$, under the conditions that the angle $\zeta_{\mathrm{CT}}$ between $\vec{m}$ and $\Delta \vec{\mu}_{\mathrm{CT}}$ is $0^{\circ}$ (expected for a MVC) ${ }^{11}$ and $\chi=90^{\circ}$ (set by experiment). ${ }^{24}$ There are many noteworthy features of the Stark spectrum in Figure 3E: the Stark spectrum of the phase-phonon band (denoted by an asterisk) is small and negative; there is a large, negative feature at a similar energy as the peak of the unperturbed IVB, and at higher energies there is a broader, less intense, positive feature.

When this analysis is repeated for the case where $\Delta \bar{v}$ equals 0 or $1200 \mathrm{~cm}^{-1}$, we get a Stark spectrum that differs from the one in Figure 3E. What is strikingly different about the case where $\Delta \bar{v}=1200 \mathrm{~cm}^{-1}$ (Figure $3 \mathrm{~F}$ ) is that the Stark spectrum has a large, positive feature with lower energy than the main negative feature. Thus, the presence or absence of this band can be a revealing indicator of the degree of energetic asymmetry of a MVC. For example, this band is quite prominent if we calculate the IVSE for values of the vibronic parameters 


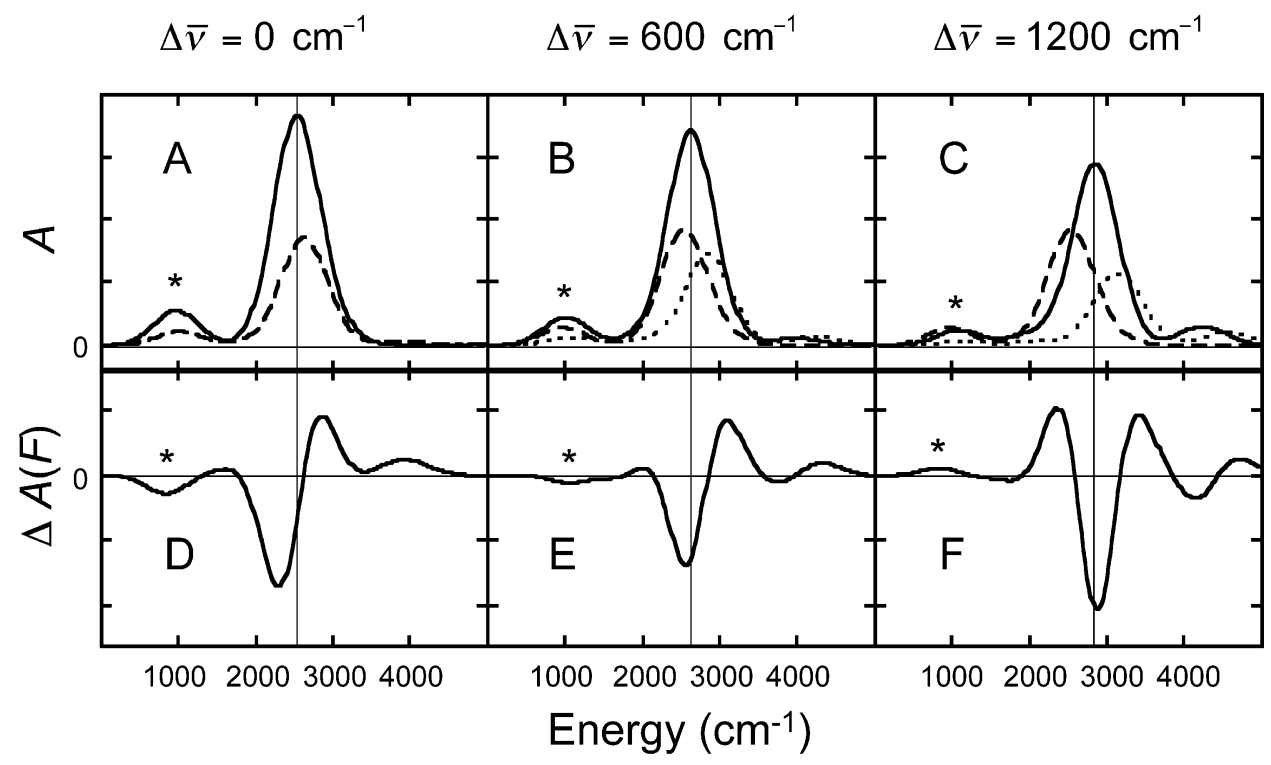

Figure 3. Absorption spectra (panels $\mathrm{A}-\mathrm{C}$ ) and Stark spectra (panels $\mathrm{D}-\mathrm{F}$ ) for ensembles of mixed valence complexes with values of $\Delta \bar{v}$ equal to 0,600 , and $1200 \mathrm{~cm}^{-1}$, respectively. Spectra are calculated as described in the text, with the value of $F \cdot f \Delta \mu_{\mathrm{CT}}$ set equal to $600 \mathrm{~cm}{ }^{-1}$. In panels $\mathrm{A}-\mathrm{C}$ we imagine an ensemble made of two oriented subpopulations and treat the transition dipole moments of these populations as scalars: the solid line corresponds to the absorption spectrum of this ensemble when there is no applied electric field; the dashed line corresponds to the absorption spectrum for the population defined by $\Delta \vec{\mu}_{\text {CT }}$ parallel to $\vec{F}$; the dotted line corresponds to the absorption spectrum for the population defined by $\Delta \vec{\mu}_{\text {CT }}$ antiparallel to $\vec{F}$. The dotted and dashed lines overlap precisely in panel A. In panels D-F we illustrate the Stark spectra calculated for an isotropic ensemble, treating the transition dipole moments for all orientational subpopulations as vectors. We note that in each case the spectra in panels $\mathrm{D}-\mathrm{F}$ have similar line shapes as would be calculated if the solid lines in panels $\mathrm{A}-\mathrm{C}$ were subtracted from the sum of the dashed and dotted lines in these panels. In each panel the asterisk indicates either the phase-phonon band (panels A-C) or its Stark effect (panels D-F).

suggested by Johnson et al. ${ }^{27}$ and even more prominent if we use the values that were proposed by Reimers and Hush when the observed dipole strength of the IVB of $\mathrm{P}^{+}$was weighted preferentially to the value of $\% L$ in their global analysis of experimental data (not shown). ${ }^{6}$ What is noticeably different about the case where $\Delta \bar{v}=0 \mathrm{~cm}^{-1}$ (Figure 3D) is that the large, negative band in the IVSE peaks at a lower energy than the peak of the IVB. Thus, the presence or absence of this offset can also be a revealing indicator of the degree of energetic asymmetry of a MVC.

These same observations can be restated by employing the classical Stark analysis to approximate each IVSE as a composition of the zeroth, first, and second derivatives of its corresponding IVB. ${ }^{24}$ Although the effect of the field on the line shape of the IVB of each individual MVC violates a principal assumption behind the classical Stark analysis, this discrete parametrization still provides a useful characterization of a continuous line shape. Whereas a classical Stark analysis would reveal a positive second-derivative component to account for most of the IVSE line shape when $\Delta \bar{v}=1200 \mathrm{~cm}^{-1}$, it would reveal this component to contribute negligibly to the IVSE line shape when $\Delta \bar{v}=0 \mathrm{~cm}^{-1}$ (not shown). In the case where $\Delta \bar{v}$ $=600 \mathrm{~cm}^{-1}$, the second-derivative component is neither insignificant nor the whole story; the IVSE also has smaller, but significant, contributions from a negative zeroth-derivative component and a negative first-derivative component (not shown).

IVBs and IVSEs calculated for values of $\bar{\nu}_{\text {anti }}$ other than 1200 $\mathrm{cm}^{-1}$ have somewhat different appearances than those illustrated in Figure 3. For example, as the value of $\bar{v}_{\text {anti }}$ is changed, the separation between the main and satellite bands of the IVB roughly track this value. As a result, when the value of $\bar{v}_{\text {anti }}$ is halved to $600 \mathrm{~cm}^{-1}$, there is a cancellation between the Stark effects of these two features in the region just above $3000 \mathrm{~cm}^{-1}$, which leads to a reduction in the size of the Stark spectrum in this region (not shown). Nevertheless, the increasing importance of the second-derivative component with increasing values of $\Delta \bar{v}$ is still observed.

One important conclusion from this discussion is that the shape of an IVSE is sensitive to changes in the value of $\Delta \bar{v}$; this shape is also sensitive to changes in the values of $V_{0}$ and $\lambda_{\text {anti }}$, as well as symmetric mode reorganization energies, as discussed in detail elsewhere. ${ }^{24}$ More importantly, a change of only a few hundred wavenumbers to $\Delta \bar{v}$ can cause much larger changes to the peak positions and line shapes of an IVSE than to those of an IVB. This suggests that IVSEs of reaction centers with mutations in the vicinity of $\mathrm{P}$, many of which have been shown to have very similar IVBs, ${ }^{27,29}$ may nevertheless display dramatically different IVSEs.

This treatment of IVSEs assumes that $\psi_{\mathrm{P}_{\mathrm{L}}}{ }^{+} \mathrm{P}_{\mathrm{M}}$ and $\psi_{\mathrm{P}_{\mathrm{L}} \mathrm{P}_{\mathrm{M}}}{ }^{+}$ interact exclusively with each other. Since we have claimed that unquantified interactions with additional electronic states may compromise the use of eqs 1 and 3 for determining $\Delta \mu_{\mathrm{CT}}$ from the dipole strength of an IVB, we must explain both why this is true and why the same interactions need not compromise the use of a two-state vibronic coupling model for determining $\Delta \mu_{\mathrm{CT}}$ from an IVSE. In support of our first claim, we note the increase in complexity which each additional electronic state would bring to our problem; for example, any relationship such as eq 1 between $\% L$ and $m^{2}$ derived from a three-state model must necessarily include more parameters than just $\Delta \mu_{\mathrm{CT}}$. This must be the case since the normalization condition used to derive eq 1 for the two-state model ${ }^{11}$ has an additional degree of freedom in a three-state model. A similar argument applies to eq 3 .

The second claim can be justified using perturbation theory using the Hamiltonian

$$
\left(\begin{array}{ll}
0 & V_{\mathrm{AB}} \\
V_{\mathrm{AB}} & \Delta \bar{v}_{\mathrm{AB}}-F \cdot f \Delta \mu_{\mathrm{AB}}
\end{array}\right)
$$

In this case the unperturbed Hamiltonian is defined by the generic basis states $\psi_{\mathrm{A}}$ and $\psi_{\mathrm{B}}$, with energies equal to 0 and 
$\Delta \bar{v}_{\mathrm{AB}}$, respectively. In the limit where the value of $\Delta \bar{v}_{\mathrm{AB}}$ is much larger than the values of both $V_{\mathrm{AB}}$ and $F \cdot f \Delta \mu_{\mathrm{AB}}$, the two eigenvalues of eq 5 are perturbed by two terms, $F \cdot f \Delta \mu_{\mathrm{AB}}$ and $V_{\mathrm{AB}}{ }^{2} / \Delta \bar{v}_{\mathrm{AB}}(F)$. The second term reduces to $V_{\mathrm{AB}}{ }^{2} / \Delta \bar{v}_{\mathrm{AB}}$ in this limit, such that the effect of $F$ on the eigenvalues is given by $F \cdot f \Delta \mu_{\mathrm{AB}}$ alone. Thus, even though $V_{\mathrm{AB}}$ does affect the eigenvalues of eq 5, it does not, in this limit, affect their field sensitivities. Similarly, according to perturbation theory, $\psi_{\mathrm{A}}$ is mixed into $\psi_{\mathrm{B}}$ with a probability amplitude equal to $V_{\mathrm{AB}} /$ $\Delta \bar{v}_{\mathrm{AB}}(F)$, which reduces to $V_{\mathrm{AB}} / \Delta \bar{v}_{\mathrm{AB}}$ in this limit. Thus, even though $V_{\mathrm{AB}}$ does affect the eigenfunctions of eq 5 , it does not, in this limit, affect their field sensitivities (or lack thereof). Since it is the eigenvalues that determine the transition energy of a spectrum, and it is the probability amplitudes that determine its dipole strength, this argument demonstrates that there exist instances for which $V_{\mathrm{AB}}$ may affect the peak position and dipole strength of a spectrum without affecting its Stark effect. These insights are readily transferable to the case of two vibronically coupled diabatic surfaces at each nuclear configuration where the energy difference between a pair of surfaces is larger than both the electronic coupling between them and the field perturbation to this energy difference.

Of course, Figure 2B illustrates that this perturbative argument is likely not relevant to the interaction between $\psi_{\mathrm{P}_{\mathrm{L}}}{ }^{+} \mathrm{P}_{\mathrm{M}}$ and

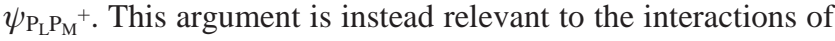
either $\psi_{\mathrm{P}_{\mathrm{L}}{ }^{+} \mathrm{P}_{\mathrm{M}}}$ or $\psi_{\mathrm{P}_{\mathrm{L}} \mathrm{P}_{\mathrm{M}}}{ }^{+}$with electronic states which may lead to intensity borrowing from the IVB. Reimers and Hush have identified a number of low-lying electronic states in special pair radical cations; ${ }^{15}$ interactions among these states and exciting radiation give rise to both a trip-doublet band at $8000 \mathrm{~cm}^{-1}$ and a second-highest-occupied molecular orbital (SHOMO) to highest-occupied molecular orbital (HOMO) transition which overlaps with the IVB. ${ }^{44}$ Since the trip-doublet band appears more than $5000 \mathrm{~cm}^{-1}$ higher in energy than the IVB, even if the electronic couplings which lead to intensity borrowing by

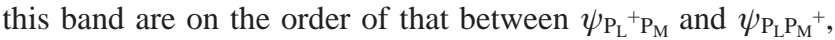
the perturbative argument will be applicable to these interactions: thus, the field sensitivities of the eigenvalues and eigenstates that give rise to the IVB should not be affected to as great an extent as the IVB itself. The SHOMO $\rightarrow$ HOMO transition has little oscillator strength compared to the IVB,

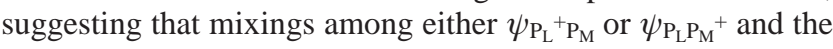
states which define this transition are weak; i.e., the relevant couplings, $V_{\mathrm{AB}}$, are small compared to the relevant energy differences, $\Delta \bar{v}_{\mathrm{AB}}$. Thus, even if $\Delta \bar{v}_{\mathrm{AB}}$ is on the order of $F \cdot$ $f \Delta \mu_{\mathrm{AB}}$, the values of $V_{\mathrm{AB}} / \Delta \bar{v}_{\mathrm{AB}}(F)$ and $V_{\mathrm{AB}}{ }^{2} / \Delta \bar{v}_{\mathrm{AB}}(F)$ should be small for most RCs in the isotropic sample, such that the field sensitivity of the IVB should not be perturbed by these terms as much as its dipole strength has been when $F=0$. Although we are not arguing that the sum of these many interactions has a negligible effect on the IVSE (in all likelihood they do lend some uncertainty to the determination of $\Delta \mu_{\mathrm{CT}}$ from the IVSE of $\mathrm{P}^{+}$), these arguments do justify the claim that $\Delta \mu_{\mathrm{CT}}$ can be estimated more reliably in this way than from the dipole strength of the IVB.

\section{Experimental Section}

Breton and co-workers have extensively characterized the light-minus-dark difference spectrum of $\mathrm{P}^{+}$in $\mathrm{RC}$ films with different degrees of hydration..$^{9,27,29}$ Their work on WT spans a region from 250 to $13500 \mathrm{~cm}^{-1}$. We focused on two contiguous windows within this region: the region between 1800 and 5500 $\mathrm{cm}^{-1}$, which contains the IVB, and the region between 1200 and $1800 \mathrm{~cm}^{-1}$, which contains the phase-phonon bands with highest energy. We used a liquid nitrogen-cooled MCT detector and a sample cell made of Ni-coated calcium fluoride windows for both regions, but at a cost in signal-to-noise ratio and fractured windows. An increased signal-to-noise ratio in the first region was obtained by using a liquid nitrogen-cooled $\mathrm{InSb}$ detector; also, many spectra in this first region were acquired using a less fragile sample cell made of Ni-coated sapphire windows.

Poly-his tagged WT Rb. sphaeroides RCs solubilized in Triton X-100 detergent and $10 \mathrm{mM}$ Tris buffer were prepared according to the method of Goldsmith ${ }^{30}$ modified as described in Treynor and Boxer. ${ }^{31}$ Measurements were performed on samples frozen rapidly at $77 \mathrm{~K}$ using a liquid nitrogen immersion cryostat. $^{32}$ Two different classes of samples were used. Wet samples of RCs were prepared from 50/50 glycerol/buffer solutions of RCs. For some wet samples this solution was mixed with an equal volume of 50/50 glycerol/buffer saturated with potassium ferricyanide in order to prepare $\mathrm{P}^{+}$by chemical oxidation. Approximately $4 \mu \mathrm{L}$ of these solutions was wicked into empty sample cells constructed around Teflon spacers. ${ }^{26}$ Such samples were roughly $30 \mu \mathrm{m}$ thick, the precise value being determined by the spacing of interference fringes of the empty sample cell at room temperature $\left(n_{\text {ref }}=1.0\right)$. Dry samples of $\mathrm{RCs}$ were prepared by depositing roughly $2 \mu \mathrm{L}$ of concentrated $\mathrm{RC}$ solution (no glycerol) onto a Ni-coated window; this solution was then quickly spread over the surface of the window with a plastic cable tie and allowed to dry on the countertop in the dark over roughly one-half hour. The resulting dried film had a good, though imperfect, optical quality. Near-infrared linear dichroism measurements on such samples suggested that detergent-solubilized RC solutions were aligned insignificantly by this procedure. We found that the surface roughness in this case precluded direct attachment of the second Ni-coated window without air gaps, leading to breakdown when the field was applied. Therefore, a dab of Dow Corning high-vacuum grease was applied to the dried film and spread using pressure from a second Ni-coated window. Dry samples constructed in this manner, without the spacer, had thicknesses which varied widely and could not be characterized in the same manner as the wet samples. Whereas wet RCs had a saturating water absorption band obscuring the region between 2800 and 3600 $\mathrm{cm}^{-1}$, dry RCs permitted this region to be studied. Optical densities of both wet and dry samples were roughly 1.0 at 800 nm.

Infrared spectra were measured with $4 \mathrm{~cm}^{-1}$ resolution using a Bruker IFS 66V/S step-scan FTIR with a globar source and a $\mathrm{KBr}$ beam splitter. Light-on spectra were recorded under illumination from a $600 \mathrm{~W}$ tungsten/halogen bulb. This light was focused onto the sample through the windows of the cryostat. To characterize the effects of illumination intensity on the spectra, the strength of this illumination was decreased by either defocusing or filtering. A germanium window was placed in front of the detector to block exciting light above 5500 $\mathrm{cm}^{-1}$. Light-minus-dark absorption difference spectra, $\Delta A$, were calculated using 64 signal-averaged scans of each spectrum.

Stark spectra were measured using both the dc and synchronized ac methods developed by Andrews and Boxer. ${ }^{33}$ Lightminus-dark Stark spectra and chemically oxidized-minusunmodified Stark spectra (double difference spectra, $\Delta \Delta A(F)$ ) were calculated using 128 signal-averaged scans of each spectrum. Moving mirror scan rates were 20 and $5 \mathrm{kHz}$ for each method, respectively; the slower scan rate for the ac method was necessary to give the $250 \mathrm{~V} / \mu$ s slew rate of the Trek 10$10 \mathrm{~B}$ voltage amplifier enough time to achieve the high voltages 


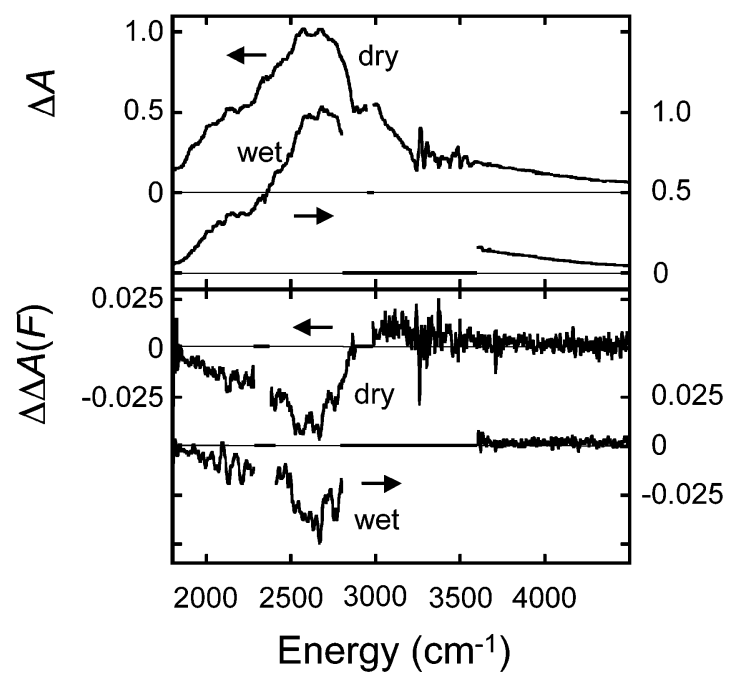

Figure 4. Light-minus-dark absorption spectra (upper panel, $\Delta A$ ) and light-minus-dark Stark spectra (lower panel, $\Delta \Delta A(F)$ ) of dry (left axis) and wet (right axis) wild-type $R b$. spheroides RCs. The spectra have been normalized and scaled to $F=1.0 \mathrm{MV} / \mathrm{cm}$ as described in the text. The region shown spans the intervalence band from 1800 to 4500 $\mathrm{cm}^{-1}$.

used in these experiments (between 0.5 and $2.1 \mathrm{kV}$ ). Although the dc method yielded better signal-to-noise ratios for a given duration of signal averaging, the spectra suffered from an irreproducible roll in the baseline over the large window of the IVSE. Stark spectra using the new synchronized ac method ${ }^{33}$ were reproducible, with no significant baseline offset.

The angle $\chi$ was set equal to $90^{\circ}$ by aligning the windows of the sample cell perpendicular to the direction of propagation of the infrared beam. When measuring Stark spectra at other values of $\chi,{ }^{16}$ an infrared polarizer was placed between the interferometer and the sample, or between the sample and the detector, to select for $s$-polarization. As a result, these spectra had much lower signal-to-noise ratios than the $\chi=90^{\circ}$ spectra.

\section{Results}

UV/vis absorption spectra of dry samples taken with a PerkinElmer Lambda 12 spectrophotometer at room temperature suggest that the principal effect of dehydration is to broaden the RC absorption bands. Also, whereas the difference spectra of wet RCs at $77 \mathrm{~K}$ are stable over many hours to the strongest illumination we have obtained, only $10 \mathrm{~min}$ of this illumination was observed to decrease the IVB intensity of dry RCs by roughly $2 \%$. On the basis of this observation, the difference absorption spectra in Figures 4 and 5 are averages of the lightminus-dark spectra acquired just before and after the $10 \mathrm{~min}$ acquisition of the light-on Stark spectrum.

The light-minus-dark absorption difference spectra at $77 \mathrm{~K}$ for dry RCs (left axis) and wet RCs (right axis) are shown offset in the upper panel of Figure 4. They have been normalized to an optical density of roughly 1.0 at their peaks for ease of comparison (the actual absorbances were roughly 0.015 and 0.030 , respectively). In both spectra, spikes at $2345 \mathrm{~cm}^{-1}$ due to the near-saturating absorbance of liquid nitrogen have been removed. For wet RCs, the saturating absorbance due to water and glycerol in the region between 2800 and $3600 \mathrm{~cm}^{-1}$ leads to extreme noisiness which has been suppressed. An exchange of deuterium oxide for water, $d_{3}$-glycerol for glycerol and phosphate for Tris buffer significantly reduced the absorption in this region but did not yield significantly improved Stark spectra (not shown). For dry RCs a number of sharp, reproduc-

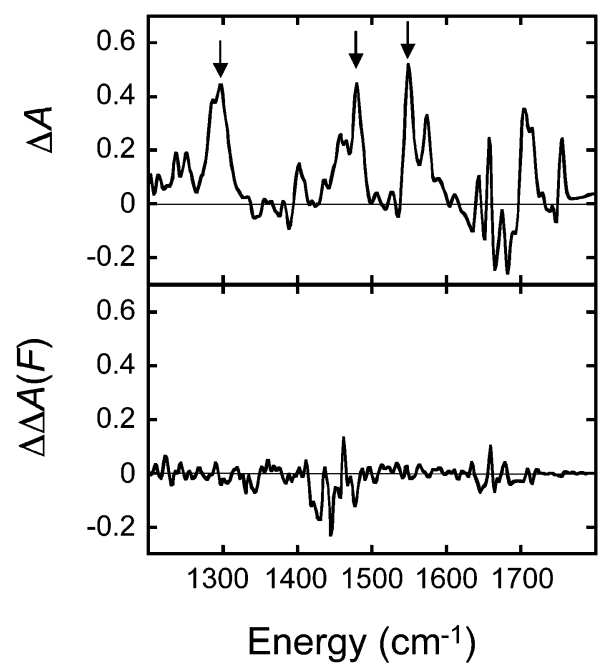

Figure 5. Light-minus-dark absorption spectrum $\Delta A$ (upper panel) and light-minus-dark Stark spectrum $\Delta \Delta A(F)$ (lower panel) of wet RCs. The spectra have been normalized as described in the text. The three phase-phonon bands with energies greater than $1200 \mathrm{~cm}^{-1}$ are labeled with arrows.

ible features are observed in the region between 3200 and 3600 $\mathrm{cm}^{-1}$; these features are likely due to changes in some $\mathrm{N}-\mathrm{H}$ and $\mathrm{O}-\mathrm{H}$ stretching frequencies upon $\mathrm{P}^{+}$formation. A spike at $2960 \mathrm{~cm}^{-1}$ is due to the saturating absorbance of the protein and vacuum grease at this frequency and has been suppressed.

The IVB of dry RCs agrees well with that published by Breton et al. for dry $\mathrm{RCs}$ at $-35^{\circ} \mathrm{C} .{ }^{34}$ Of particular note are the similar characteristics of the high-energy tail and the relative intensity of the shoulder at $2175 \mathrm{~cm}^{-1}$ to the main band at 2650 $\mathrm{cm}^{-1}$. This ratio is observed to be substantially lower for wet $\mathrm{RCs}$, in agreement with spectra recorded by Johnson et al. for RCs at $100 \mathrm{~K} .{ }^{27}$ Reimers and Hush have modeled this shoulder

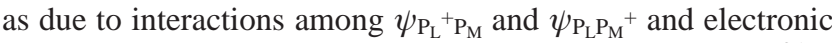
states which give rise to a $\mathrm{SHOMO} \rightarrow \mathrm{HOMO}$ transition. ${ }^{35}$

IVSEs of both dry RCs (left axis) and wet RCs (right axis) are shown offset in the lower panel of Figure $4\left(\chi=90^{\circ}\right)$. The Stark spectrum of wet RCs has been scaled to a field of $1 \mathrm{MV} /$ $\mathrm{cm}$ for ease of comparison with the literature by dividing by the square of the applied field strength, $0.72 \mathrm{MV} / \mathrm{cm}$. This field strength was calculated by dividing the voltage applied to the sample $(2.1 \mathrm{kV})$ by its thickness $(29 \mu \mathrm{m})$. This scaling is justified since the magnitude of the Stark spectra increased as the square of the applied field, and the line shape was unaffected by the applied field strength (not shown). The chemically oxidizedminus-unmodified Stark spectrum of wet RCs (not shown) has an identical line shape to that of the light-minus-dark Stark spectrum of wet RCs, implying that there is no significant contribution to the light-minus-dark Stark spectrum due to electric field effects on the population of $\mathrm{P}^{+} .36$ The sizes of these spectra suggest that more than half of RCs contribute to both types of double-difference spectra under optimized conditions.

Because the field strength of the dry RC samples was difficult to characterize due to the large uncertainty in the sample thickness and because the Stark spectra of wet and dry RCs were similar in the regions where they were readily comparable, the Stark spectrum for the dry RCs has been scaled by setting its peak amplitude to roughly the same value as the peak amplitude of the Stark spectrum of wet RCs. In this way we can get around the limitation of the uncertainty in the field strength for the dry sample. ${ }^{37}$ Whereas for dry RCs the IVSE line shape could be obtained over the entire region plotted in 
Figure 4, for wet RCs the region between 2800 and $3600 \mathrm{~cm}^{-1}$ is extremely noisy and has been suppressed. Smaller regions of noise centered at 2345 and $2960 \mathrm{~cm}^{-1}$ have also been suppressed. Polarizing the probing light beam reduces the probe intensity and yielded Stark spectra with significantly more noise than those in Figure 5; the changes in the Stark spectra with the value of $\chi$ (eq 6) were consistent with $\zeta_{\mathrm{CT}}=0^{\circ}$ (not shown).

At lower energies, the IVSEs of both wet and dry samples have a broad, negative feature with peak amplitude near 2650 $\mathrm{cm}^{-1}$. The Stark spectrum of dry RCs reveals the only zerocrossing of this IVSE near $2850 \mathrm{~cm}^{-1}$ and a weak, but significant, positive feature with a peak near $3100 \mathrm{~cm}^{-1}$. Similar results as those illustrated in Figure 4 were obtained upon normalization for different conditions of illumination intensity and sample concentration.

Figure 5 shows regions of the light-minus-dark absorption spectrum (upper panel) and the light-minus-dark Stark spectrum (lower panel) which include the phase-phonon bands highest in energy. These spectra, for wet RCs, have been scaled as in Figure 4. In agreement with the work of Breton et al., ${ }^{29}$ four major positive bands with similar intensity are visible in the light-minus-dark absorption spectrum at roughly 1300, 1475, 1550 , and $1700 \mathrm{~cm}^{-1}$. The first three bands have been assigned as phase-phonon bands, whereas the fourth is thought to be the upshifted half of a complex carbonyl bandshift. ${ }^{29}$ The increased noise in the Stark spectrum at roughly 1450 and $1650 \mathrm{~cm}^{-1}$ is due to the near-saturating absorbance of the amide bands from the protein at these energies. There do not appear to be any features in the Stark spectrum that can be assigned to the phasephonon bands themselves.

\section{Discussion}

Figure 6 illustrates the results of a classical Stark analysis of the observed IVB and IVSE of $\mathrm{P}^{+}$. The upper panel compares the IVB of dry RCs (line) and the result of applying a SavitskyGolay smoothing filter to this spectrum (open circles). The filter used is calculated for a fourth-order polynomial and includes the points within $500 \mathrm{~cm}^{-1}$ to either side of the point in question. The spikes at 2345 and $2960 \mathrm{~cm}^{-1}$ were removed so as to reduce their effects on the results of this analysis. The middle panel of Figure 6 compares the IVSE of dry RCs (line) and the linear least-squares fit (open circles) to these data using the zeroth, first, and second derivatives of the smoothed IVB (lower panel).

This fit using the classical Stark analysis is good, though imperfect. For one, there are oscillations in the fit that correspond to regions of high curvature in the absorption spectrum, especially at the junction between the shoulder and the main band, and the region from 3200 to $3600 \mathrm{~cm}^{-1}$, where the true shape of the IVB is obscured by other features in the light-minus-dark spectrum of the dry RCs. Another noticeable shortcoming of this fit is that it crosses zero at roughly 3400 $\mathrm{cm}^{-1}$ and has small negative values at higher energies, in contrast to the small positive values that are observed in this tail region. Although Born-Oppenheimer violation in the case of $\mathrm{P}^{+}$leads us to expect an imperfect fit using the classical Stark analysis, these shortcomings may be attributed in part to treating the main band and shoulder as having the same Stark parameters; however, we note that the fit in the region of the shoulder and main band is actually quite good, suggesting that the two bands do indeed have similar Stark spectra.

The derivative components of the fit from the classical Stark analysis are illustrated in the lower panel of Figure 6. Consistent with the predictions from the classical Stark analysis of the simulated spectra in panels B and E of Figure 3, none of the zeroth-, first-, or second-derivative components of this fit are

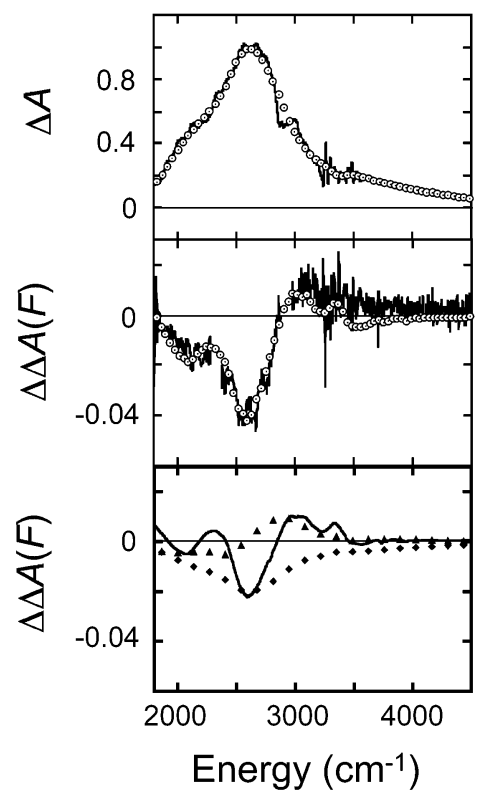

Figure 6. Classical Stark analysis (eqns 5 and 6) of spectra for dry RCs. The upper panel illustrates the intervalence band of dry RCs (line) and the result of applying the Savitsky-Golay smoothing filter described in the text (open circles). The middle panel illustrates the intervalence band Stark effect of dry RCs (line) and the best fit to these data using a sum of the zeroth, first, and second $\bar{v}$-weighted derivatives of the smoothed intervalence band (open circles). The lower panel illustrates the contributions to this fit from the zeroth, first, and second derivatives (solid diamonds, solid triangles, and solid line, respectively).

negligible, and the coefficients of these components are negative, negative, and positive, respectively. The fit value of $\Delta \mu$ is 8.2 $\mathrm{D} / f$. Also consistent with the simulation in Figure $3 \mathrm{E}$, but inconsistent with the other simulations described above, the observed IVSE has only two main features: a negative feature at a similar position as the peak position of the IVB and a broader, positive feature to higher energy. Thus, there is extensive qualitative agreement between the line shapes of the observed IVSE and that predicted using values previously estimated by Reimers and Hush from a fit to both the IVB and $\% L=70 .{ }^{638}$ In contrast, and as expected, the fit from the classical Stark analysis is inconsistent with the two-state electronic coupling model of IVSEs: ${ }^{13}$ given the fit value of $\Delta \mu$ and the estimate of $5 \mathrm{D}$ for the transition dipole moment, ${ }^{9}$ eqs $6,7,17$, and 18 in ref 13 would have predicted opposite signs than observed for both the coefficients of the zeroth and first derivatives.

We use the same single-mode vibronic coupling model to estimate the value of $\Delta \mu_{\mathrm{CT}}$ by minimizing the sum of the squared residuals between the observed and calculated IVSEs. Using the estimated values of $V_{0}=1100 \mathrm{~cm}^{-1}, \Delta \bar{v}=600 \mathrm{~cm}^{-1}, \lambda_{\text {anti }}$ $=1100 \mathrm{~cm}^{-1}, \bar{v}_{\text {anti }}=1200 \mathrm{~cm}^{-1}$, and $F=1.0 \mathrm{MV} / \mathrm{cm}$, and scaling the calculated IVB to an optical density of 1.0 at its peak, the best fit value of $\Delta \mu_{\mathrm{CT}}$ is $32 \mathrm{D} / f$. Figure 7 overlays the IVB and IVSE of dry RCs (line) with those calculated in panels $\mathrm{B}$ and $\mathrm{E}$ of Figure 3 for $F=1.0 \mathrm{MV} / \mathrm{cm}, \Delta \mu_{\mathrm{CT}}=32 \mathrm{D} / f$, and thus $F \cdot f \Delta \mu_{\mathrm{CT}}=540 \mathrm{~cm}^{-1}$ (open circles). Consistent with the observed field dependence of the IVSE, we have verified that the line shape of the calculated IVSE is independent of $F \cdot f \Delta \mu_{\mathrm{CT}}$ for values less than or equal to this value and that the magnitude of the calculated IVSE increases with the square of $F \cdot f \Delta \mu_{\mathrm{CT}}$ within this range (not shown).

Because the value of $\bar{\nu}_{\text {anti }}$ has not been constrained by previous work, we investigate how the fit value of $\Delta \mu_{\mathrm{CT}}$ depends on the value of this parameter. Given the constraint of the peak position 


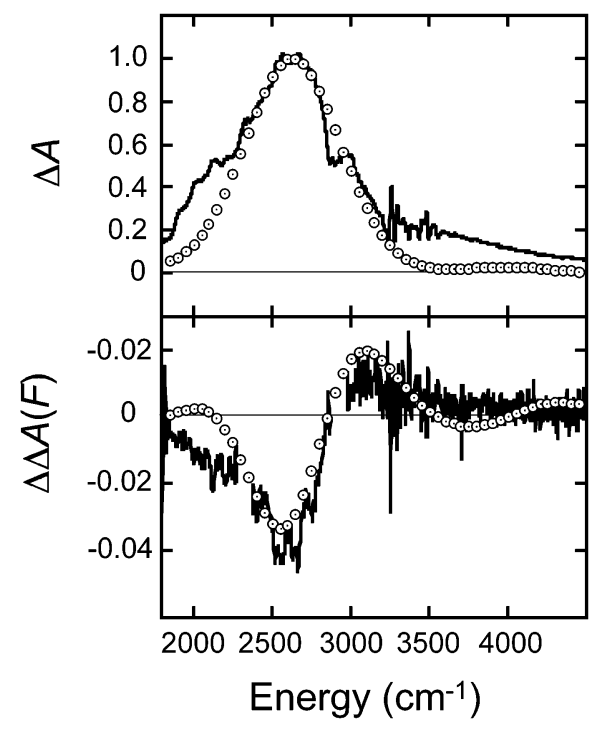

Figure 7. Fit to IVB (upper panel) and IVSE (lower panel) of dry RCs (line) using a two-state vibronic coupling model with a single antisymmetric mode (open circles). The fit has been calculated using $V_{0}=1100 \mathrm{~cm}^{-1}, \Delta \bar{v}=600 \mathrm{~cm}^{-1}, \lambda_{\text {anti }}=1100 \mathrm{~cm}^{-1}, \bar{v}_{\text {anti }}=1200$ $\mathrm{cm}^{-1}$, and $\Delta \mu_{\mathrm{CT}}=32 \mathrm{D} / f$.

of the observed IVB, we find that the fit values of $\Delta \mu_{\mathrm{CT}}$ are in the range $31-36 \mathrm{D} / f$ when $\bar{v}_{\text {anti }}$ is varied between 400 and 1600 $\mathrm{cm}^{-1}$ and $V_{0}$ is varied between 1000 and $1200 \mathrm{~cm}^{-1}$. For example, if the value of $\bar{\nu}_{\text {anti }}$ is halved to $600 \mathrm{~cm}^{-1}$, the amplitude of the positive feature at $3100 \mathrm{~cm}^{-1}$ is decreased as described earlier, leading to an increase in the fit value of $\Delta \mu_{\mathrm{CT}}$ to $35 \mathrm{D} / f$ (not shown); since this change in $\bar{v}_{\text {anti }}$ also induces a noticeable shift to the IVB, in this case we have calculated the fit value of $\Delta \mu_{\mathrm{CT}}$ having increased the value of $V_{0}$ to $1200 \mathrm{~cm}^{-1}$ to offset this shift. Since the IVB and IVSE line shapes are affected only slightly when $\lambda_{\text {anti }}$ is modified by $200 \mathrm{~cm}^{-1}$, we have repeated this analysis for this range of reorganization energies and have found that the range of fit values for $\Delta \mu_{\mathrm{CT}}$ is extended to 30$36 \mathrm{D} / f$. Modifications of $\Delta \bar{v}$ by even $100 \mathrm{~cm}^{-1}$ significantly decrease the agreement between the observed and calculated IVSE line shapes, and so we have not calculated fit values of $\Delta \mu_{\mathrm{CT}}$ in these cases. This important observation suggests that the Stark line shape should be very sensitive to mutations that differentially perturb the energies of $\psi_{\mathrm{P}_{\mathrm{L}}}{ }^{+} \mathrm{P}_{\mathrm{M}}$ and $\psi_{\mathrm{P}_{\mathrm{L}} \mathrm{P}_{\mathrm{M}}}{ }^{+}$. This has been observed in several RC mutants that selectively perturb the energies of $\psi_{\mathrm{P}_{\mathrm{L}}}{ }^{+} \mathrm{P}_{\mathrm{M}}$ or $\psi_{\mathrm{P}_{\mathrm{L}} \mathrm{P}_{\mathrm{M}}}$ by inserting or removing hydrogen bonds to $\mathrm{P}$ and will be reported separately. ${ }^{39}$

Using $\Delta \mu_{\mathrm{CT}}=36 \mathrm{D} / f$, the Stark spectrum for the single phasephonon band in panels $\mathrm{B}$ and $\mathrm{E}$ of Figure 3 is calculated to be -0.03 times as large as the phase-phonon band itself. Calculations with two antisymmetric modes (not shown) demonstrate that the size of this multiplier decreases when the total reorganization energy is distributed between the $1200 \mathrm{~cm}^{-1}$ mode and a mode with a different frequency. These small multipliers are consistent with the data in Figure 5: if the phasephonon bands in the upper panel are multiplied by 0.03 , the result is roughly 4 times smaller than the noise level in the observed Stark spectrum. To determine experimentally the true value of this multiplier, we would need to collect data with a field strength that is at least twice as large as we have applied or with at least 16 times more signal averaging. Since the many phase-phonon bands observed for $\mathrm{P}^{+}$imply that its reorganization energy is divided among many modes, ${ }^{34}$ it is likely that an even greater improvement in signal-to-noise would be required to see the phase-phonon band Stark effect of $\mathrm{P}^{+}$.
The similarities between the observed and calculated IVSE line shapes are apparent in Figure 7, as are some notable differences, which provide a useful basis for discussing how more complex vibronic coupling models would be expected to affect the fit value of $\Delta \mu_{\mathrm{CT}}$, given the value of $\% L$ and other constraints. ${ }^{40}$ Since the largest percentage discrepancies between the observed and calculated IVBs and IVSEs in Figure 7 occur in the shoulder and tail regions of the spectra, it is reasonable to focus on these regions. The classical Stark analyses of the observed and calculated spectra have already answered this question with regard to the IVSE line shape. First, these analyses have shown that the observed IVSE in the shoulder region behaves in the same way as it does for the main band; thus, if one imagined superimposing a copy of the calculated IVB upon itself, shrunken and shifted to fill out the observed intensity in the shoulder region, superimposing a copy of the calculated IVSE, shrunken and shifted in the same way, would also improve the fit to the observed IVSE in this region. Second, although we did not expect the classical Stark analysis to return a perfect fit to the observed IVSE line shape, it did demonstrate that much of that line shape could be accounted for by the zeroth, first, and second derivatives of the observed IVB line shape; thus, if additional complexity could broaden the calculated IVB in this tail region, it should improve the fit to the observed IVSE line shape by smoothing out the oscillations calculated for this region.

In both of these cases, the purpose of the additional complexity in the vibronic coupling model would be, in a sense, to broaden the calculated IVB, thus broadening the features in the calculated IVSE. Because broader absorption spectra yield smaller derivative spectra, we would expect the additional broadness to increase the fit value of $\Delta \mu_{\mathrm{CT}}$ from the value of $32 \mathrm{D} / f$. However, since we have shown that the zeroth-derivative component makes a substantial contribution to the IVSE in this case (see Figure 6), additional broadness in the calculated IVB will not affect the size of the calculated IVSE to as great an extent as it might if the calculated IVSE were dominated by either a first- or second-derivative line shape. This point also suggests that little error has been introduced to the magnitude of the IVSE of dry RCs by scaling it to the well-calibrated magnitude of the IVSE of wet RCs.

To better quantify the effect of additional model complexity on the fit value of $\Delta \mu_{\mathrm{CT}}$, we determined fit values of $\Delta \mu_{\mathrm{CT}}$ using vibronic coupling models that incorporate either two antisymmetric modes or one antisymmetric mode and one symmetric mode. ${ }^{24}$ Given the constraints of the peak position of the IVB and the strong mixing between $\psi_{\mathrm{P}_{\mathrm{L}}}{ }^{+} \mathrm{P}_{\mathrm{M}}$ and $\psi_{\mathrm{P}_{\mathrm{L}} \mathrm{P}_{\mathrm{M}}}{ }^{+}$, the models with two antisymmetric modes do not significantly increase the quality of the fit to the rest of the IVB in either the shoulder or tail regions (not shown). As mentioned above, similar-looking IVBs can lead to different-looking IVSEs when the vibrational frequencies of the modes are varied, but the range of fit values of $f \Delta \mu_{\mathrm{CT}}$ is not altered by the additional model complexity in this case.

Even small symmetric mode reorganization energies can lead to significant asymmetric broadening of the IVB, as illustrated in the upper panel of Figure 8. This IVB was calculated using $V_{0}=1100 \mathrm{~cm}^{-1}, \Delta \bar{v}=600 \mathrm{~cm}^{-1}, \lambda_{\text {anti }}=1100 \mathrm{~cm}^{-1}, \bar{v}_{\text {anti }}=$ $1200 \mathrm{~cm}^{-1}, \lambda_{\text {sym }}=125 \mathrm{~cm}^{-1}$, and $\bar{\nu}_{\text {sym }}=800 \mathrm{~cm}^{-1}$; the value of the full width at half-maximum of the Gaussian broadening was $650 \mathrm{~cm}^{-1}$. The best fit to the observed IVSE in this case was calculated with $\Delta \mu_{\mathrm{CT}}=32 \mathrm{D} / f$ and is illustrated in the lower panel of Figure 8. Whereas the effects of the symmetric mode reorganization energy on the IVB appear substantial in 


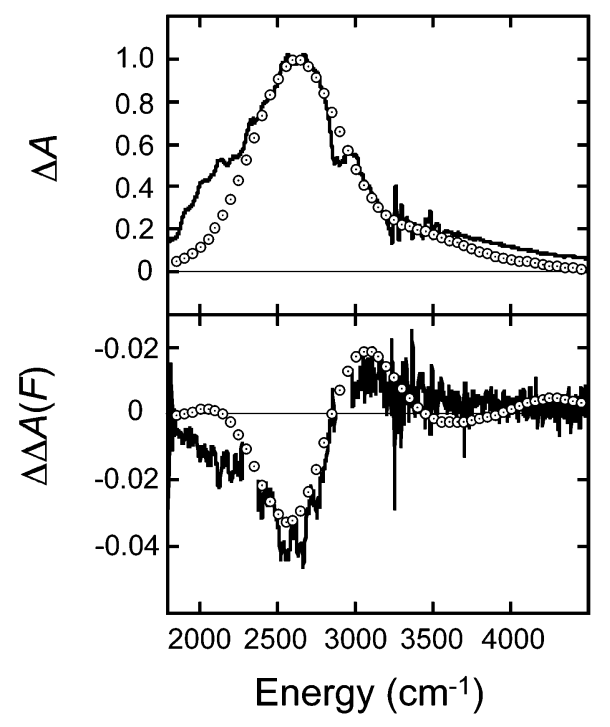

Figure 8. Fit to IVB (upper panel) and IVSE (lower panel) of dry RCs (line) using two-state vibronic coupling model with a single antisymmetric mode and a single symmetric mode (open circles). The fit has been calculated using $V_{0}=1100 \mathrm{~cm}^{-1}, \Delta \bar{v}=600 \mathrm{~cm}^{-1}, \lambda_{\text {anti }}=$ $1100 \mathrm{~cm}^{-1}, \bar{v}_{\text {anti }}=1200 \mathrm{~cm}^{-1}, \lambda_{\text {sym }}=125 \mathrm{~cm}^{-1}, \bar{v}_{\text {sym }}=800 \mathrm{~cm}^{-1}$, and $\Delta \mu_{\mathrm{CT}}=32 \mathrm{D} / f$.

the wing region, the effects on the main band are small; since the largest changes to the IVB are localized to a region of relatively low intensity, it is not surprising that the effects of the symmetric mode reorganization energy on the IVSE line shape are subtle, and the effect on the fit value of $\Delta \mu_{\mathrm{CT}}$ is insignificant. Thus, we conclude that the fit value of $\Delta \mu_{\mathrm{CT}}$ is within the range $30-36 \mathrm{D}$. Taking into account an uncertainty in the applied field strength due to the $1-2 \mu \mathrm{m}$ uncertainty in the wet sample thickness, we report the value of $\Delta \mu_{\mathrm{CT}}$ as $33 \pm$ $5 \mathrm{D} / f$. Of course, this result is qualified by the use of only two electronic states in the vibronic coupling model we have used. In this regard it is important to note that, using a model that incorporates the two additional electronic states which give rise to the SHOMO $\rightarrow$ HOMO transition, Reimers and Hush have demonstrated that the IVSE of $\mathrm{P}^{+}$can be fit well using a value of $\Delta \mu_{\mathrm{CT}}$ equal to $31 \mathrm{D}$, a value based on $\mathrm{B} 3 \mathrm{LYP} / 3-21 \mathrm{G}$ calculations of this parameter. ${ }^{45}$

Assuming that $f$ equals either 1.0 or 1.3 , the $33 D$ value of $f \Delta \mu_{\mathrm{CT}}$ corresponds to an effective charge-transfer distance of either 6.9 or $5.3 \AA$, respectively; this range of values is both shorter than the $8.0 \AA$ distance between the magnesium ions of $\mathrm{P}^{+}$and longer than the $2.3 \AA$ value which was estimated using eq 1 with $\% L=70$ and $m^{2}=25 \mathrm{D}^{2}$. Using the values of 6.9 and $5.3 \AA$ with the estimate of 70 for $\% L$ and eq 1 , the total dipole strength of the calculated IVB and phase-phonon band in Figure 3B would be either 230 or $140 \mathrm{D}^{2}$, respectively. For comparison, the $8.0 \AA$ distance between the magnesium ions of $\mathrm{P}^{+}$would lead to a dipole strength of $310 \mathrm{D}^{2}$. Thus, the effective charge-transfer distance is reduced from $8.0 \AA$ by as much as one-third of this value, resulting in a reduction of the expected dipole strength from $310 \mathrm{D}^{2}$ by as much as one-half of this value. Ligand polarizabilities, metal-ligand delocalization, and other effects have been invoked to explain such reductions in MVCs. ${ }^{13}$

Since the observed dipole strength of the IVB is only $25 \mathrm{D}^{2}$, this result might imply that intensity borrowing by other transitions in $\mathrm{P}^{+}$-containing $\mathrm{RC}$ reduces the dipole strength of the IVB by more than $100 \mathrm{D}^{2}$. It is interesting to note in this regard that the ratio of integrated positive intensity to integrated negative intensity is substantially larger for both of the calculated
IVSEs in Figures 7 and 8 than for the observed IVSE. This observation implies that the transition moment of the IVB of $\mathrm{P}^{+}$is more sensitive to the value of $\Delta \bar{v}$ than is expected from the two-state model we have used. This extra sensitivity could be indicative of significant intensity borrowing by higher-lying electronic states which increases when the IVB is shifted to higher energy.

Alternatively, both observations may indicate that factors besides $\Delta \mu_{\mathrm{CT}}$ and $\% L$ make significant contributions to the dipole strength of an IVB even in the two-state case. $100 \mathrm{D}^{2}$ is such a large amount of intensity borrowing that one would expect to see obvious manifestations of this intensity throughout the near-infrared and visible parts of the spectrum, yet the dipole strength of the trip-doublet band near $8000 \mathrm{~cm}^{-1}$ is only $7 \pm 2$ $\mathrm{D}, 2,9,15$ and the intensities of transitions due to the monomeric pigments in RCs are not observed to change much upon oxidation of $\mathrm{P},{ }^{9,41}$ If in fact there exists a significant shortcoming in our understanding of the dipole strength of IVBs, the results here suggest that Stark spectroscopy and the method of analysis we have employed to determine the value of $\Delta \mu_{\mathrm{CT}}$ will be important tools for investigating the nature and significance of these factors. Revisiting published Stark data ${ }^{13,17-20}$ with this new method ${ }^{24}$ could provide a large number of preliminary results in this regard.

Acknowledgment. T.P.T. is supported by a predoctoral fellowship from the Fannie and John Hertz Foundation and a Stanford Graduate Fellowship. This work was supported by grants from the NSF Biophysics and Chemistry Programs. The FTIR used for this work is located at the Stanford FEL Center supported by the Air Force Office of Scientific Research (Grant F49620-00-1-0349). We thank Jeff Reimers and Noel Hush for very helpful comments.

\section{References and Notes}

(1) Deisenhofer, J.; Norris, J. R. The Photosynthetic Reaction Center Academic Press: San Diego, CA, 1993; Vol. 1.

(2) Reimers, J. R.; Hutter, M. C.; Hughes, J. M.; Hush, N. S. Int. J. Quantum Chem. 2000, 80, 1224.

(3) Demadis, K. D.; Hartshorn, C. M.; Meyer, T. J. Chem. Rev. 2001, $101,2655$.

(4) Lendzian, F.; Huber, M.; Isaacson, R. A.; Endeward, B.; Plato, M.; Bonigk, B.; Mobius, K.; Lubitz, W.; Feher, G. Biochim. Biophys. Acta 1993, $1183,139$.

(5) Mattioli, T. A.; Williams, J. C.; Allen, J. P.; Robert, B. Biochemistry 1994, 33, 1636.

(6) Reimers, J. R.; Hush, N. S. Chem. Phys. 1996, $208,177$.

(7) For compactness we have adopted the following notation conventions: (i) if a symbol is used in both subscripted and unsubscripted forms, then the unsubscripted symbol refers to the general class of the subscripted symbols; (ii) if a symbol such as $m$ is used as both $m$ and $\vec{m}$, then $m$ alone refers to the norm of $\vec{m},|\vec{m} \cdot \vec{m}|^{1 / 2}$; (iii) if a symbol such as $\Delta \bar{v}$ is used as both $\Delta \bar{v}$ and $\Delta \bar{v}(F)$, then $\Delta \bar{v}$ alone refers to the $F$-independent component of $\Delta \bar{v}(F)$.

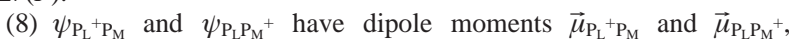

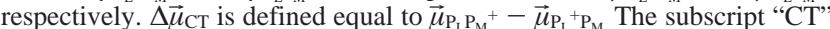
stands for charge transfer. 7503.

(9) Breton, J.; Nabedryk, E.; Parson, W. W. Biochemistry 1992, 31,

(10) Mantele, W. G.; Wollenweber, A. M.; Nabedryk, E.; Breton, J. Proc. Natl. Acad. Sci. U.S.A. 1988, 85, 8468.

(11) Equation 1 is straightforward to derive by combining the expression for the transition dipole moment ${ }^{13} \vec{m}=c_{1} c_{2} \Delta \vec{\mu}_{\mathrm{CT}}$ with a formal definition of $\% L$ as $c_{1}^{2} \times 100$, where $c_{1}$ and $c_{2}\left(c_{1}^{2}+c_{2}^{2}=1\right)$ are the respective coefficients of $\psi_{\mathrm{P}_{\mathrm{L}}{ }^{+} \mathrm{P}_{\mathrm{M}}}$ and $\psi_{\mathrm{P}_{\mathrm{L}} \mathrm{P}_{\mathrm{M}}}{ }^{+}$in the ground adiabatic electronic state which results from their interaction. When using a vibronic coupling model, we estimate the value of $\% L$ at the nuclear configuration corresponding to the minimum of the ground adiabatic surface.

(12) Ermler, U.; Fritzsch, G.; Buchanan, S. K.; Michel, H. Structure 1994, 2, 925.

(13) Brunschwig, B. S.; Creutz, C.; Sutin, N. Coord. Chem. Rev. 1998, 177,61 . 
(14) Parson, W. W.; Nabedryk, E.; Breton, J. Mid- and Near-IR Electronic Transitions of P+: New Probes of Resonance Interactions and Structural Asymmetry in Reaction Centers. In The Photosynthetic Bacterial Reaction Center II; Breton, J., Vermeglio, A., Eds.; Plenum Press: New York, 1992; p 79.

(15) Reimers, J. R.; Hush, N. S. Inorg. Chim. Acta 1994, 226, 33. 213.

(17) Oh, D. H.; Boxer, S. G. J. Am. Chem. Soc. 1989, 111, 1130. 6880 .

(19) Karki, L.; Hupp, J. T. J. Am. Chem. Soc. 1997, 119, 4070.

(20) Bublitz, G. U.; Laidlaw, W. M.; Denning, R. G.; Boxer, S. G. J. Am. Chem. Soc. 1998, 120, 6068.

(21) The ground and excited states, $\psi_{1}$ and $\psi_{2}$, have dipole moments

$\vec{\mu}_{1}$ and $\vec{\mu}_{2}$, respectively. $\Delta \vec{\mu}_{12}$ is defined equal to $\vec{\mu}_{2}-\vec{\mu}_{1}$.

(22) Reimers, J. R.; Hush, N. S. J. Phys. Chem. 1991, 95, 9773.

(23) Piepho, S. B.; Krausz, E. R.; Schatz, P. N. J. Am. Chem. Soc. 1978, $100,2996$.

(24) Treynor, T. P.; Boxer, S. G. J. Phys. Chem. A, submitted.

(25) Treynor, T. P.; Boxer, S. G., manuscript in preparation.

(26) Andrews, S. S.; Boxer, S. G. J. Phys. Chem. A 2000, 104, 11853

(27) Johnson, E. T.; Muh, F.; Nabedryk, E.; Williams, J. C.; Allen, J. P.; Lubitz, W.; Breton, J.; Parson, W. W. J. Phys. Chem. B 2002, 106, 11859.

(28) Since we have conducted a coarsely grained, single-mode calculation, the calculation is expected to show oscillations where a multimode calculation might show a smoother line shape; when one imagines the region between 3000 and $4000 \mathrm{~cm}^{-1}$ to be smoothed, it is more clear why we refer to Figure $2 \mathrm{C}$ as broader than either Figure 2A or Figure 2B.

(29) Nabedryk, E.; Allen, J. P.; Taguchi, A. K. W.; Williams, J. C.; Woodbury, N. W.; Breton, J. Biochemistry 1993, 32, 13879.

(30) Goldsmith, J. O.; Boxer, S. G. Biochim. Biophys. Acta 1996, 1276, 171 .

(31) Treynor, T. P.; Ishii, C. Y.; Boxer, S. G., manuscript in preparation.

(32) Andrews, S. S.; Boxer, S. G. Rev. Sci. Instrum. 2000, 71, 3567.

(33) Andrews, S.; Boxer, S. G. Appl. Spectrosc. 2001, 55, 1161.

(34) Breton, J.; Nabedryk, E.; Clerici, A. Vibr. Spectrosc. 1999, 19, 71

(35) Reimers, J. R.; Hush, N. S. J. Chem. Phys. 2003, 119, 3262.

(36) Boxer, S. G.; Goldstein, R. A.; Lockhart, D. J.; Middendorf, T. R.; Takiff, L. J. Phys. Chem. 1989, 93, 8280.
(37) While this manuscript has been in preparation, improvements in the experimental method have determined the dry sample thickness to an accuracy of $15 \% .{ }^{39}$ Preliminary results using the improved method support the scaling we have performed here, but they have yet to achieve a comparable signal-to-noise ratio.

(38) When Reimers and Hush ( $\mathrm{RH}$ ) used a vibronic coupling model with a single antisymmetric mode to determine the values of $V_{0}, \Delta \bar{\nu}$, and $\lambda_{\text {anti, }}$, their model was not the same as the PKS model ${ }^{6}$ however, as discussed elsewhere ${ }^{24}$ the two models are the same in the limit of strong mixing. Using the values of $V_{0}, \Delta \bar{v}$, and $\lambda_{\text {anti }}$ which they have proposed for $\mathrm{P}^{+}$, we have found that the only noticeable difference between the IVB calculated using the PKS model and the IVB calculated using the RH model is that the latter is slightly more asymmetric. We find this difference to be insignificant, and so we retain their fit values of these parameters for our investigation. While this manuscript was being completed, Reimers and Hush submitted for publication a multimode treatment of the IVB of $\mathrm{P}^{+}$ based on yet another vibronic coupling model ${ }^{35}$ Using this new multimode model, Reimers and Hush have refined the values of $V_{0}$ and $\Delta \bar{v}$ to be 1000 and $550 \mathrm{~cm}^{-1}$, respectively. Based on the results reported here, these differences are insignificant, so we retain the original values of these parameters as obtained using the single-mode model.

(39) Kanchanawong, P.; Treynor, T. P.; Boxer, S. G., manuscript in preparation.

(40) As discussed elsewhere, because of Born-Oppenheimer violation it is unclear how best to calculate the dipole strength for some IVBs. ${ }^{24,42}$ Thus, for some simulations (not shown) we have added a correction term to the calculation of the transition dipole as suggested by Wong and Schatz, ${ }^{24,43}$ but we have not been able to improve the overall quality of the fit to the IVB and IVSE in this manner. For other simulations (not shown) we have added a linear field dependence to $V_{0},{ }^{24}$ but we have not been able to improve the overall quality of the fit to the IVSE in this manner.

(41) Moss, D. A.; Leonhard, M.; Bauscher, M.; Mantele, W. G. FEBS Lett. 1991, 283, 33

(42) Talaga, D. S.; Zink, J. I. J. Phys. Chem. 1996, 100, 8712.

(43) Wong, K. Y.; Schatz, E. Prog. Inorg. Chem. 1981, 28, 369.

(44) Reimers, J. R.; Shapley, W. A.; Hush, N. S. J. Chem. Phys. 2003, $119,3240$.

(45) Reimers, J. R.; Hush, N. S., manuscript in preparation. 